\title{
REDESCUBRIENDO A UN FILÓSOFO HÍBRIDO: GEORGES CANGUILHEM
}

\author{
Francisco Vázquez García \\ Universidad de Cádiz \\ francisco.vazquez@uca.es
}

Recibido: 26 diciembre 2013; Aceptado: 7 enero 2014.

Cómo citar este artículo/Citation: Vázquez García, Francisco (2014), "Redescubriendo a u filósofo híbrido: Georges Canguilhem", Asclepio 66 (2): p065. doi: http://dx.doi.org/10.3989/asclepio.2014.29

FERTÉ, Louise, JACQUARD, Aurore y VERMEREN, Patrice (dir.). La formation de Georges Canguilhem. Un entredeux-guerres philosophique, Paris, Hermann Éditeurs, 2013, 373 pp. [ISBN: 978-2-7056-8666-6]

ROTH, Xavier. Georges Canguilhem et l'unité de l'expérience. Juger et agir (1926-1939), Paris, Vrin, 2013, 247 pp. [ISBN: 978-2-7116-2491-1]

La obra del filósofo y médico francés Georges Canguilhem (Castelnadaury 1904- Marly le Roy 1995) conoce en la actualidad un extraordinario revival. Este se produce tanto a escala nacional francesa como internacional, y con un radio interdisciplinar, involucrando a especialistas de las más diversas materias (genetistas, ecólogos, neurocientíficos, biotecnólogos, médicos, sociólogos, psicólogos, historiadores de las ciencias, filósofos). Este despegue del interés se inició poco antes de su fallecimiento, y queda testimoniado en la multiplicación de coloquios sobre su pensamiento, monografías en forma de libros o de números de revista, traducciones de sus escritos a diversas lenguas y centros de investigación y documentación que llevan su nombre (Le Blanc, 2003, p. 9; Debru, 2004, pp. 28-29).
Hasta mediados de la década de 1990, Canguilhem era considerado un filósofo relevante pero "menor" (Bouveresse, 2011, p. 8), destacando por el magisterio que ejerció sobre pensadores más conocidos, como Michel Foucault, Pierre Bourdieu o Louis Althusser y su círculo. Más que un filósofo en el pleno sentido de la palabra, se le consideraba como un autor con una obra relativamente reducida, muy confinada en el terreno específico de la historia de la medicina y de las ciencias de la vida, encuadrado en la denominada "escuela de epistemología histórica francesa" (Cavaillés, Koyré, Bachelard, Foucault). Los estudios existentes, poco numerosos hasta los años noventa, lo presentaban como heredero del tipo de historia de las ciencias forjada por Gaston Bachelard (a quien sucedió en 1955 como director del Institut d'Histoire des Sciences

Copyright: (c) 2014 CSIC. Este es un artículo de acceso abierto distribuido bajo los términos de la licencia Creative Commons Attribution-Non Commercial (by-nc) Spain 3.0. 
et des Techniques de la Sorbonne) y como maestro de Michel Foucault, cuya tesis de Estado dirigió en 1961. Su propia contribución como epistemólogo e historiador de la medicina y la biología, quedaba así un tanto desdibujada entre el análisis bachelardiano de las ciencias físicas y químicas, y los estudios "arqueogenealógicos" de Foucault sobre las ciencias humanas.

Esta situación comenzó a cambiar radicalmente a partir de 1994. En esa fecha, la editorial neoyorkina Zone Books publicó una extensa antología de textos suyos (Delaporte, 1994), dando así a conocer su obra al público anglosajón, pues hasta entonces sólo se habían vertido al inglés dos textos de Canguilhem, On the normal and the pathological (1978) e Ideology and rationality in the history of life sciences (1998). De hecho, el público norteamericano, cuya tradición en epistemología estaba dominada por la filosofía analítica, importada a través de Wittgenstein y de los exiliados del Círculo de Viena, ${ }^{1}$ sólo se había interesado por Canguilhem a través del magisterio que este había ejercido sobre Michel Foucault, cuyos textos conocían un verdadero "boom" en Estados Unidos, desde la década de los ochenta. Pero lo novedoso de esa antología es que contenía una completísima bibliografía crítica sobre Canguilhem, realizada por su discípulo Camille Limoges.

Pues bien, en esa bibliografía se incluía a la referencia a más de 100 trabajos de Canguilhem, publicados entre 1926 y 1939, la mayoría artículos de revista y recensiones (en algunos casos firmados con seudónimo), pero también tres libros, uno de ellos un manual (Traité de logique et de morale, 1939, redactado junto a Camille Planet); en los otros casos se trata de escritos breves. El primero, redactado junto a Félicien Challaye, vio la luz en 1932, con el título La paix sans aucune réserve. El segundo fue encargado en 1935 por el Comité de Vigilances des Intellectuels Antifascistes y se tituló Le fascisme et les paysans. La casi totalidad de ese corpus había pasado desapercibida para la crítica, que rutinariamente databa hasta entonces la primera obra de Canguilhem en 1943, su tesis de medicina, defendida en la Universidad de Strasbourg, replegada en Clermont Ferrand durante la ocupación. Se titulaba Essai sur quelques problémes concernant le normal et le pathologiqie. El propio Canguilhem, por otro lado, siempre había guardado silencio sobre esa primera etapa intelectual suya. Se ponía así al descubierto un "Canguilhem perdido" (Braunstein, 2011), un "Canguilhem antes de Canguilhem" (Braunstein, 2000), de modo que la que se estimaba como su opera prima era en realidad una investigación de madurez.

La bibliografía publicada por Camille Limoges puso sobre la pista a toda una serie de investigadores, empezando por Jean François Braunstein, y continuan- do con una larga serie de estudiosos (Yves Schwartz, Elisabeth Roudinesco, Dominique Lecourt, Pierre Macherey, Xavier Roth, François Dagognet, François Delaporte, Guillaume Le Blanc, Claude Debru, CamiIle Limoges, Gilles Renard, Guillaume Pénisson, Cyryaque Ebissienine $)^{2}$ que, desde la segunda mitad de los noventa, han iniciado una reinterpretación a fondo del legado filosófico de Georges Canguilhem. Este proceso culminó recientemente con el inicio de la publicación de sus Oeuvres Complètes (5 volúmenes previstos), por la editorial Vrin. El primer tomo, que vio la luz en 2011, contiene precisamente ese "corpus de juventud", Écrits philosophiques et politiques 1926-1939, con la inclusión de varias introducciones y estudios realizados por algunos de los especialistas que se han mencionado.

Todas estas iniciativas desplegadas a raíz del descubrimiento del "Canguilhem perdido", han llevado a replantear por completo el significado de su obra. Canguilhem ya no es tenido en cuenta simplemente como un historiador de la biología y la medicina, ni siquiera como un epistemólogo de estas disciplinas. Hoy se le considera como un filósofo en el pleno sentido de esta palabra, de modo que, no sólo se pone al descubierto su pretensión de fundar una antropología filosófica (Saint-Sernin, 1996, Le Blanc, 2002, Debru, 2004) a partir de las disciplinas biomédicas, sino su vocación de filósofo práctico. Es decir, los aspectos morales y políticos (e incluso estéticos) aparecen como los elementos nucleares de su programa filosófico, desde un fuerte compromiso con los valores universales de la justicia y la igualdad.

El análisis de este dilatado itinerario juvenil, hasta hace poco desconocido y que llega prácticamente a la cuarentena de Canguilhem, se ha llevado a cabo en dos trabajos recientes. El primero es una obra colectiva, publicada en 2013, que recoge los trabajos presentados en el Coloquio "Un entre-deux-guerres philosophique: la formation de Georges Canguilhem", celebrado en París VIII, el 14 y 15 de junio de 2012. EI segundo recoge la tesis doctoral de Xavier Roth sobre la génesis del pensamiento de Canguilhem, recientemente editada por Vrin.

En el primer caso se recoge casi una veintena de trabajos realizados por estudiosos procedentes de los cinco continentes. Su denominador común es la exploración de los escritos del Canguilhem joven, evitando al mismo tiempo la tentación de explicar toda la obra posterior del filósofo partiendo de esa etapa inicial.

Los coordinadores del volumen distribuyen las distintas intervenciones en seis grandes secciones temáticas. La primera ("Philosopher") incluye dos aporta- 
ciones (Macherey, Vauday), dedicadas a dilucidar los rasgos de la actividad filosófica en el joven Canguilhem. Macherey insiste en que la filosofía constituye para Canguilhem una tarea normativa, no destinada a producir verdades sino a considerar el valor de la verdad (la ciencia) en relación con otros valores (la técnica, el arte, la moral, etc). La instancia desde la que se ejerce ese examen normativo sería para el primer Canguilhem, formado en el idealismo kantiano de sus maestros (Alain, Lagneau), el espíritu. Pero desde finales de la década de los treinta, este sería reemplazado por la vida. Aquí se entiende el perfil híbrido del filosofar de Canguilhem, no centrado en pensar a partir de los grandes autores de la tradición filosófica, sino del material suministrado por saberes ajenos a la Historia de la Filosofía. Vauday, por su parte, subraya las coincidencias tempranas de filosofía y medicina en el joven Canguilhem. En ambos casos se trata, no de enunciar grandes leyes explicativas, sino de diagnosticar, de evaluar condiciones siempre singulares.

La segunda sección ("Pacifisme et Résistance") se dirige más bien hacia la trayectoria política del pensador de Castelnaudury. ¿Cómo explicar su tránsito, mediada la década de 1930, desde el pacifismo de Alain hacia el compromiso con la Resistencia? Renzi Ragghianti indaga las bases del pensamiento político de Alain y rastrea su dilatada presencia en los textos del primer Canguilhem. Georges Navet, por otro lado, muestra de qué modo una filosofía rebelde contra la sumisión a los hechos, en línea con Alain, pero al mismo tiempo sensible respecto a las singularidades históricas, frente al esencialismo de Alain, condujo a Canguilhem hacia la Resistencia.

La tercera sección ("Le Penseur et le Saltimbanque") incluye una serie de colaboraciones que conectan las decisiones políticas del primer Canguilhem con su temple filosófico como formador del juicio. Laurence Cornu relaciona el viraje intelectual del joven pensador, matriculándose en la Facultad de Medicina cuando era profesor de filosofía en un liceo de Toulouse, con su inflexión política, renunciando al pacifismo. En ambos casos se constata una toma de partido por las fuerzas que resisten a la muerte, una opción por la vida humana, concretada en el compromiso médico y antifascista. Emmanuel Péhau muestra de qué modo el servicio militar funcionó en Canguilhem como una experiencia crucial, que a contrario, le puso al descubierto las exigencias de una vida realmente humana, afrontada como conquista de uno mismo mediante la forja del propio juicio. Didier Moreau cierra este apartado con un artículo que desentraña con minuciosidad la pedagogía filosófica del Canguilhem temprano. Esta apuntaba a elevar la experiencia humana hacia el ideal de justicia, más allá de toda apelación a una naturaleza dada en el hombre.
La cuarta sección ("Contre le culte du fait: critique de la psychologie et des sciences humaines") contiene un conjunto de aportaciones centradas en el diálogo del joven Canguilhem con las ciencias del hombre, y en particular en su confrontación con la psicología. Jean François Braunstein, uno de los mejores conocedores de la obra canguilhemiana, reconstruye, mediante el estudio de los textos de juventud, la génesis de los argumentos de Canguilhem (epistémicos y morales) frente a la psicología del comportamiento. Se recomponen así las continuidades y deslizamientos que conducirán a las influyentes intervenciones de madurez, de 1956 y 1980 respectivamente, donde el filósofo francés se despacha contra esta disciplina. Alejandro Bilbao, por su parte, pone de manifiesto cómo el psicoanálisis, a diferencia de la psicología conductista, gozaba de la estima de Canguilhem, cuya antropología de lo negativo no dejaba de coincidir con las aportaciones freudianas. Rachid Dehdouh insiste en este mismo asunto, trayéndolo a la actualidad. Hoy en día, el psicoanálisis y la psicología cognitiva escaparían a la crítica canguilhemiana, que ve en la psicología una "escuela de sumisión a los hechos". Aurore Jacquard da término a este apartado contrastando las críticas de la psicología realizadas respectivamente por Canguilhem y Lacan. Ambas parecen abrir un mismo modo de pensar la subjetividad, situado más allá de la psicología introspectiva y del conductismo.

El quinto apartado ("Les valeurs de la vie, la médécine, la biologie") se sitúa ya en el momento del desplazamiento canguilhemiano desde el idealismo hacia el vitalismo. Mazarine Pingeot enfatiza la importancia del artículo de Canguilhem sobre Descartes y la técnica (1937), tanto para la interpretación de la obra cartesiana como para la elaboración de una filosofía que recalca la precedencia de la técnica sobre la ciencia. Cristina López, por su lado, evoca a Foucault para explicar por qué el vitalismo de Canguilhem rompe los márgenes de la filosofía del sujeto. Esta constituye un obstáculo para comprender los nexos que vinculan a la vida misma, en sus bases moleculares, con el conocimiento biológico. Lucie Rey se retrotrae sin embargo a la obra del cirujano René Leriche, mostrando, más allá de las críticas de las que fue objeto por parte de Canguilhem, la filiación spinozista de ambos pensadores. Elena Donato, finalizando este bloque, en uno de los trabajos más interesantes del volumen, reconstruye la teoría canguilhemiana de la creación, insinuada ya en la década de 1930, y pone al descubierto una subyacente estética en el pensamiento de Canguilhem.

Los ensayos que componen la sexta y última sección ("Canguilhem, historien des sciences"), lidian ya con problemas que nos devuelven la imagen de un Canguilhem más familiar, epistemólogo e his- 
toriador de las ciencias. Todos ellos relacionan los planteamientos canguilhemianos con los de autores próximos. Pierre Cassou-Nogués, por ejemplo, considera que las tesis de Estado y complementaria, defendidas por Jean Cavaillès en 1938, inauguran esa radical conversión histórica del kantismo, que la propia epistemología de Canguilhem prosiguió, con sus propios medios, en el ámbito de las ciencias de la vida. François Delaporte traslada la discusión desde Cavaillès hasta Foucault. Polemiza con la lectura de Canguilhem efectuada por Étienne Balibar. Según este, la diferencia foucaultiana entre "estar en la verdad" y "decir la verdad", deforma, para uso propio, lo que habían sugerido Koyré y Canguilhem a propósito de Galileo. Delaporte desmiente a Balibar; Foucault comprendió y se atuvo perfectamente al distingo que realizó su maestro. Abundando en la pista foucaultiana, Monique David-Ménard encuentra el eje que vincula el quehacer filosófico de Canguilhem y de su discípulo de Poitiers, con la epistemología kantiana. Del mismo modo que el pensador de Königsberg, sus modernos secuaces franceses articulan el filosofar a partir de un doble movimiento: el gesto escéptico, poniendo al descubierto las ilusiones con las que debe romper la razón para constituirse, y el gesto crítico, trazando los límites dentro de los cuales conceptos (Canguilhem) y enunciados (Foucault) funcionan dibujando una forma de racionalidad específica.

Aunque todos los estudios reunidos en el volumen comparten el mismo interés por redefinir el alcance filosófico de Canguilhem a partir de la interpretación de su obra de juventud, queda patente la dispersión temática que preside el conjunto. Esto no sucede en la monografía publicada por Xavier Roth. Producto de una tesis doctoral defendida en 2010, bajo la tutela de Claude Debru y Camille Limoges, en su investigación se asiste a la gestación de Canguilhem como uno de los grandes filósofos del siglo XX.

En efecto, Canguilhem se ocuparía de los problemas clásicos de la filosofía, pero esa condición de filósofo quedaría encubierta por los medios intelectuales utilizados, sustentados en estudios de historia de la ciencia y epistemología, sumamente circunscritos y específicos. De ahí lo enigmático de una obra aparentemente modesta que ha influido decisivamente en pensadores de la talla de Foucault, Bourdieu o Althusser; lo sorprendente de un autor ocupado con asuntos teóricos muy especializados y poco dado a efusiones sobre las vivencias y el compromiso, que sin embargo se involucró hasta los tuétanos en la Resistencia. De ahí también lo chocante de una reflexión sobre la medicina que, pese a remitir a un saber biomédico periclitado en muchos sentidos (el de las décadas de 1940 y 1950), sigue conservando una extraordinaria vigencia y actualidad.
Semejante condición paradójica es el punto de partida del trabajo de Roth. Este introduce el problema realizando una introducción general a la filosofía de Canguilhem. Esta se identifica en lo esencial con una historia de las ciencias asentada a su vez en una filosofía de la medicina que afronta la vida como creación de valores. El cometido del libro consiste en trazar la génesis de este concepto vertebral; el de "normatividad biológica". Sin negar las fuentes biomédicas alemanas de esta noción en Canguilhem (Goldstein sobre todo, pero también Uexküll, Herxheimer, Von Weiszacker, Jaspers), se insiste en que el encuentro fecundo con la tradición teutónica sólo fue posible a partir de la previa formación filosófica recibida.

Se tratará entonces de restaurar esa herencia filosófica viendo cómo la categoría de "normatividad vital" y por extensión, todo el proyecto teórico de Canguilhem, se arraigan y a la vez se separan de aquélla. Ese legado en el que se formó Canguilhem es el del neokantismo francés, conformado principalmente en torno a la dinastía compuesta por Lachelier, Lagneau y Alain. No se trata sólo de unos textos, sino de todo un "estilo de pensamiento" (Fleck), lo que se conoce como el "análisis reflexivo". En la primera parte de su estudio, Roth delimita los perfiles y los jalones de este estilo, a través de un conocimiento exhaustivo de la producción juvenil de Canguilhem, tanto la publicada como la inédita, y de la difícil familiaridad con las obras de sus antepasados teóricos. Analiza así el modo en que la conversión antropológica de la epistemología kantiana marcó decisivamente las disposiciones filosóficas de Canguilhem.

Lagneau y Alain impulsaron un filosofar emplazado en la estela de Kant, que consistía en preguntarse, ante toda experiencia dada (perceptiva, cognitiva, técnica, artística, etc.), cuáles eran sus condiciones trascendentales de posibilidad. Estas quedaban fundadas, en último término, sobre la actividad sintética del espíritu, identificada con la facultad de juzgar, esto es, con la posición de valor que unifica lo dado y que está presente, según estos autores, ya en el nivel de la percepción. Alain le dio a la analítica trascendental kantiana un giro decididamente moral. El juicio denotaba la dignidad del sujeto, cuyo empeño era la ordenación, esto es, la evaluación de una materia de suyo caótica e indiferente. Se consagraba así la oposición entre el espíritu y el mundo. Todo el énfasis en la condición normativa del quehacer filosófico, toda la rebelión frente al determinismo de cualquier índole, presentes en Canguilhem, encuentran aquí su raíz. Roth efectúa, con minuciosidad de orfebre, la reconstrucción de los vericuetos conceptuales y personales que vinculaban al joven Canguilhem con esta herencia del kantismo francés. 
Tras esta primera parte en la que se da cuenta de la filiación de Canguilhem con sus maestros kantianos y se ofrecen las primeras pistas sobre sus desplazamientos respecto a este legado, se abre un capítulo de transición. En este se despliega una visión sintética del cambio experimentado en la trayectoria de Canguilhem. Este trastocamiento le llevaría a separarse del idealismo kantiano de formación sin dejar por ello de conservar, como instancias permanentes, algunos de sus motivos inspiradores. Una clave para comprender este cambio la suministra el comentario entusiasta de Canguilhem publicado a raíz de la aparición de Les mots et les choses (1966), de Michel Foucault.

En ese texto, el maestro parecía advertir en el libro del discípulo una explicación profunda de su propio tránsito desde el idealismo kantiano hasta el vitalismo. En el decurso de la episteme moderna, las condiciones de posibilidad de la experiencia dejaban de consistir en la unidad sintética de la apercepción, esto es, en la actividad juzgadora de un sujeto pensante, de un Cogito. Entre mediados de la década de 1930 y en el transcurso del siguiente decenio, Canguilhem llegó a identificar las condiciones a priori de la experiencia, ya no con el sujeto, sino con la vida afrontada como creación de valores. El Cogito dejaba así su lugar a un trascendental objetivo y empírico, la Vida, un proceso que Foucault había examinado en la mencionada obra, a escala del pensamiento occidental.

Pues bien, tras esta suerte de flashward que nos hace saltar hasta 1966, la segunda parte de la monografía de Roth se dedica a recomponer paso a paso, delimitando cuidadosamente sus distintas etapas, ese proceso de ruptura de Canguilhem respecto a su herencia kantiana. En realidad se trata de un desplazamiento, pues el problema nuclear se mantiene constante, y no es otro que el de la unidad de la experiencia. ¿Qué es lo que unifica la experiencia y sus distintos registros valorativos? El retrato que se efectúa de esta evolución dista de ajustarse a una imagen lineal. Si Lagneau había mostrado que el entendimiento como facultad teórica se subordinaba al juicio como decisión evaluadora, Canguilhem iba a subrayar, tras el largo rodeo reflexivo de los años treinta sobre la precedencia de la técnica y la creación, que la medicina constituye un saber normativo, sustentado en el acto de juzgar, que se corresponde a su vez con la propia actividad normativa del viviente.

Este viraje del entendimiento al juicio y del juicio a la acción, tiene también su vertiente práctico-política. Canguilhem fue un fiel seguidor del pacifismo de Alain, pero la nueva deriva hacia lo concreto -que el filósofo de Castelnaudary compartía con su unidad generacional, hacia la primacía de la acción respecto a los principios teóricos, le llevó a desarrollar una sen- sibilidad por las singularidades históricas de la que su maestro carecía. Aquí se sitúan el interés y a la vez las reticencias de Canguilhem respecto al marxismo, que llegaron a su momento más intenso en 1935, con la publicación de Le fascisme et les paysans, y que Roth examina con mucho detenimiento. Aquí se localiza, asimismo, la proximidad vivida por Canguilhem en Toulouse, con los republicanos españoles y su experiencia del fascismo y la guerra. Esto sería decisivo, según Roth, no sólo en la biografía política de Canguilhem, con la renuncia al pacifismo, sino también en el modo de plantear el problema de la unidad de la experiencia. Aquí se entroniza también el encuentro con Cavaillès y la decisión de involucrarse activamente en la Resistencia.

En su exposición, Roth considera cruciales para marcar la distancia de Canguilhem con Alain, las reflexiones del primero sobre la técnica y la creación, publicadas en los últimos años treinta y comienzos de los cuarenta. La técnica, guiada por exigencias vitales, implica desbordar la voluntad de verdad dictada por el entendimiento; se funda en ficciones, en errores que sólo ex post facto serán denunciadas por el pensamiento científico. Esta prioridad del error y de la ficción era inadmisible en el programa filosófico de Alain y del análisis reflexivo. El paso siguiente consistirá en identificar la vida y sus necesidades ("exigencias del viviente") como raíz de ese movimiento de construcción y destrucción de ficciones, que en último término caracterizará a la historia de las ciencias biológicas. En esta pendiente, Canguilhem se encontrará con la epistemología bachelardiana y su primado del "error", la "rectificación" y la "ruptura epistemológica". Por otro lado, la identificación de la "vida" como actividad sintética ordenadora de la experiencia cobrará forma en el concepto clave de "normatividad vital".

Roth reconstruye con rigor y meticulosidad esta travesía, deslindando los principales debates, las convergencias y las divergencias, revisando algunos tópicos (la relación del tronco Alain-Canguilhem con el vitalismo de Bergson) y polemizando sobre otros (poniendo en tela de juicio la sobredimensionada influencia de Goldstein, de Marx o de Nietzsche sobre Canguilhem). Se trata sin duda de una historia internalista, centrada en delimitar las filiaciones y las transformaciones conceptuales (sobre todo en el eje constituido por Lagneau, Alain y Canguilhem), pero que no deja de evocar la relación de éstas con las alteraciones en la biografía política de Canguilhem y con la atmósfera cultural y social del momento. Sería no obstante conveniente completar el estudio histórico-conceptual de Roth con un estudio sociogenético que explorara a fondo la formación del habitus filosófico de Canguilhem en el entrecruzamiento de los universos sociales e intelectuales, al modo en que Moreno Pestaña (2006) lo hizo con Foucault. Pero esa es otra tarea. 
Los dos textos comentados pueden servir de acicate para una recuperación hispánica de la obra de Canguilhem. De hecho ya existen algunos signos del renovado interés por su obra en los países de lengua española. Desde hace unos pocos años, la editorial bonaerense Amorrortu, está publicando las principales obras de madurez del filósofo, aún no vertidas en nuestra lengua, ${ }^{3}$ y comienza también a proliferar la literatura secundaria. En nuestro país, quitando el caso aislado de algunos historiadores de la medicina (Laín Entralgo y sobre todo Felipe Cid), este autor sólo empezó a interesar a partir de la década de los 70, leído desde la exégesis que hacían de él los discípulos del filósofo marxista Louis Althusser como forjador de una epistemología de bases materialistas. Posteriormente, las referencias a Canguilhem han venido sobre todo de parte de los comentaristas españoles de la obra de Foucault, dado el eco extraordinario que ha conocido en nuestro país el pensamiento de este último. Curiosamente, una filosofía como la

\section{NOTAS}

1 Sobre la recepción británica y norteamericana de Canguilhem, véase respectivamente Gordon, 1998 y Grene, 2000. Sobre la recepción alemana, véase Schöttler, 2012.

2 Esto no confina la existencia de eminentes conocedores de la obra de Canguilhem al mundo francés. Conocidos filósofos anglonorteamericanos como los estadounidenses Paul Rabinow y Arnold Davidson, el canadiense lan Hacking 1998 o el británico Nikolas Rose, han publicado trabajos sobre Canguilhem. Aparte de esto hay que mencionar los trabajos de estudiosos italianos (Cammelli, Bianco, Cutro, Paltrinelli) y alemanes (Mühle, Deuber-Mankowski) sobre su obra.

\section{BIBLIOGRAFÍA}

Bouveresse, Jacques (2011), "Préface aux Oeuvres Complètes de Georges Canguilhem". En: Canguilhem, Georges, Oeuvres Complètes. Écrits philosophiques et politiques 1926-1939, Paris, Vrin, pp. 7-69.

Braunstein, Jean François (2000), "Canguilhem avant Canguilhem", Revue d'histoire des sciences, 53 (1), pp. 9-26.

Braunstein, Jean François (2011), “Introduction. Á la découverte d'un Canguilhem perdu". En: Canguilhem, Georges, Oeuvres Complètes. Écrits philosophiques et politiques 1926-1939, Paris, Vrin, pp. 101-137.

Debru, Claude (2004), Georges Canguilhem, Science et no science, Paris, Éditions Rue d'Ulm.

Delaporte, François (ed.) (1994), A vital rationalist. Selected writings from Georges Canguilhem, New York, Zone Books. de Canguilhem, identificada explícitamente con un "vitalismo racionalista", que tantas similitudes guarda, en su filosofía de la vida como "aventura", de la técnica, del perspectivismo, de la ética "deportiva" (Braunstein, 2011: 118) y en su antropología, con el "raciovitalismo" de Ortega y con la filosofía médica de Laín Entralgo (Montiel, 2008), no ha sido hasta ahora estudiada en España por sí misma. Ya es hora de que esto cambie.

\section{AGRADECIMIENTOS}

Este trabajo se ha realizado gracias a la financiación de la Dirección General de Investigación del Ministerio de Ciencia e Innovación, dentro del proyecto "Vigilancia de fronteras, colaboración crítica y reconversión: un estudio comparado de la relación de la filosofía con las ciencias sociales en España y Francia (1940-1990)", referencia FFI2010-15196 (subprograma FISO).

3 Escritos sobre la medicina (2004), Ideología y racionalidad en las ciencias de la vida (2005) y Estudios de historia y de filosofía de las ciencias (2009). Anteriormente estaban disponibles Lo normal y lo patológico (Buenos Aires, Siglo XXI, 1971, a partir de la edición francesa de 1966); La formación del concepto de reflejo en los siglos XVII y XVIII (Barcelona, Avance, 1975, hoy de difícil localización) y El conocimiento de la vida (Barcelona, Anagrama, 1976, a partir de la edición francesa de 1965).

Gordon, Colin (1998), "Canguilhem: life, health and death", Economy and Society, 27 (2-3), pp. 182-189.

Grene, Marjorie (2000), "The philosophy of science of Georges Canguilhem: a transatlantic view", Revue d'histoire des sciences, 53 (1), pp. 47-64

Le Blanc, Guillaume (2002), La vie humaine. Anthropologie et biologie chez Georges Canguilhem, Paris, PUF.

Le Blanc, Guillaume (2003), "Présentation”. En: Le Blanc, G. (dir.), Lectures de Canguilhem. Le normal et le pathologique, Paris, ENS Éditions, pp. 9-16.

Montiel, Luis (2008), “Actualité de la philosophie de la médecine de G. Canguilhem, P. Laín et E. Rothschuh dans la formation des médecins". En: Fagot-Largeault, Anne, Debru, Claude, Morange, Michel, Han, Hee Jin (eds.), Philosophie et Médecine. En hommage á Georges Canguilhem, Paris, Vrin, pp. 203-219. 
Moreno Pestaña, José Luis (2006), En devenant Foucault. Sociogénèse d'un grand philosophe, París, Éditions du Croquant.

Saint-Sernin, Bertrand (1996), "Une anthropologie biologique et historique", Cahiers Philosophiques, 69, pp. 57-65.
Schöttler, Peter (2012), "Sur la réception de 'l'épistémologie française' en Allemagne". En: AAVV., Epistemology and History from Bachelard and Canguilhem tot today history of science, Berin, Max-Planck-Institut für Wissenschaftsgeschichte, pp.41-46. 